Trauma Berufskrankh 2009 · 11[Suppl 1]:107-111 DOI 10.1007/s10039-008-1407-8

Online publiziert: 5. Juni 2008

๑) Springer Medizin Verlag 2008

\author{
A. Seekamp \\ Klinik für Unfallchirurgie (Traumatologie), \\ Universitätsklinikum Schleswig-Holstein, Campus Kiel
}

\title{
Fraktur am proximalen Femur
}

\section{Frakturform und Risiken}

dern in der Regel eine operative Versorgung. Bei richtiger Klassifikation und entsprechend gestellter Operationsindikation sowie richtiger Wahl des Implantats wird diese in der Regel unkompliziert durchzuführen sein, sodass man an sich von einem einfachen Fall ausgehen kann. Aber allein die Klassifikation ist bereits für das Outcome sehr entscheidend.

\section{Frakturtypisierung}

\section{Klassifikationen}

Die heute gängigste Frakturklassifikation ist die der Arbeitsgemeinschaft für Osteosynthesefragen (AO) ( $\bullet$ Abb. 1), bei welcher insgesamt 3 Hauptgruppen unterschieden werden. Innerhalb jeder Hauptgruppe werden dann nochmals 3 Untergruppen klassifiziert. Wesentliches Unterscheidungsmerkmal sind einerseits der Frakturverlauf in Beziehung zur Trochanterebene und andererseits, inwieweit der Bruch auch den Trochanter minor betrifft. Ist Letzterer ebenfalls frakturiert, wird davon ausgegangen, dass die mediale Abstützung fehlt, und es wird von einer so genannten instabilen proximalen Femurfraktur gesprochen.

Auch in anderen Klassifikationssystemen, wie der Einteilung nach Evans [3] aus dem Jahre 1949 oder auch der nach Jensen u. Michaelsen [5] aus dem Jahr 1975, hat die Unterscheidung in stabile und instabile Frakturen eine wesentliche Bedeutung. Gleichermaßen wird in diesen Klassifikationssystemen das Kriterium der Instabilität mit der begleitenden Fraktur des Trochanter minor in Beziehung gebracht.
Aufgrund der anatomischen Verhältnisse handelt es sich bei den Brüchen des proximalen Femurs um so genannte extrakapsuläre Frakturen im Gegensatz zu den intrakapsulären Frakturen, die ausschließlich den Schenkelhals betreffen. Die Therapie Letzterer beruht auch auf der Wiederherstellung einer mechanischen Stabilität, die Ausheilung selbst ist aber in erster Linie durch die Biologie bzw. die Durchblutung des Femurkopfs bestimmt. Nicht selten kommt es gerade bei medialen Schenkelhalsfrakturen im weiteren Verlauf zu einer so genannten Femurkopfnekrose.

Bei den extrakapsulären Frakturen, wie der inter- und der subtrochanteren Femurfraktur, basiert die Behandlung ebenfalls auf der Wiederherstellung der mechanischen Stabilität, allerdings ist auch die Ausheilung in erster Linie durch deren Erhalt bestimmt. Wichtig in diesem Zusammenhang ist der Verlauf der so genannten Zug- und Drucktrabekel im Bereich des Schenkelhalses. Mechanisch günstig ist dessen Valgisierung, da hierbei die Kraft in einem relativ stumpfen Winkel zur Frakturebene von proximal nach distal eingeleitet wird. Soweit möglich kann es daher sinnvoll sein, im Rahmen der operativen Versorgung eine leichte Valgisierung im Bereich der Fraktur herbeizuführen. Durch die Belastungsdynamik am proximalen Femur kommt es in der Regel zu einer Varisierung im Bereich der Fraktur und bei gleichzeitiger Instabilität der medialen Kortikalis (begleitende Fraktur des Trochanter minor) auch zu einer Medialisierung des Schafts. Bezüglich der Analyse der Fraktur sei daher nochmals betont, dass insbesondere die mediale Abstützung beurteilt werden muss.

\section{Therapie}

\section{Verfahren}

Bei der Wahl des operativen Verfahrens müssen, neben der Fraktur selbst, auch die Erwartungen des Patienten berücksichtig werden. In der Regel gilt, dass es diesem ermöglicht werden sollte, möglichst rasch in sein gewohntes Lebensumfeld zurückzukehren und seine vorbestehende körperliche Leistungsfähigkeit wieder zu erreichen. Bei älteren Patienten kann dies bedeuten, dass sie lediglich wieder im Gehwagen mobilisiert werden können. In jedem Fall aber gilt, dass man unmittelbar postoperativ wieder eine Vollbelastung anstreben muss. Bei jüngeren Patienten kann dies eher bedeuten, dass sie ihre volle sportliche Leistungsfähigkeit wieder erreichen möchten.

\section{Technik}

Die operative Taktik beinhaltet in der Regel eine geschlossene Reposition der Fraktur nach Länge, Achse und Rotation.

Der Patient wird auf dem Extensionstisch gelagert. Bei entsprechender Kontrolle unter Bildwandler sollte daher unter leichtem Längszug, Abduktion und Innenrotation des Femurschafts eine nahe$\mathrm{zu}$ anatomische Reposition möglich sein. Der Trochanter minor muss nicht anatomisch reponiert werden, da er in der Regel auch bei größerer Dislokation wieder knöchernen Anschluss findet. 

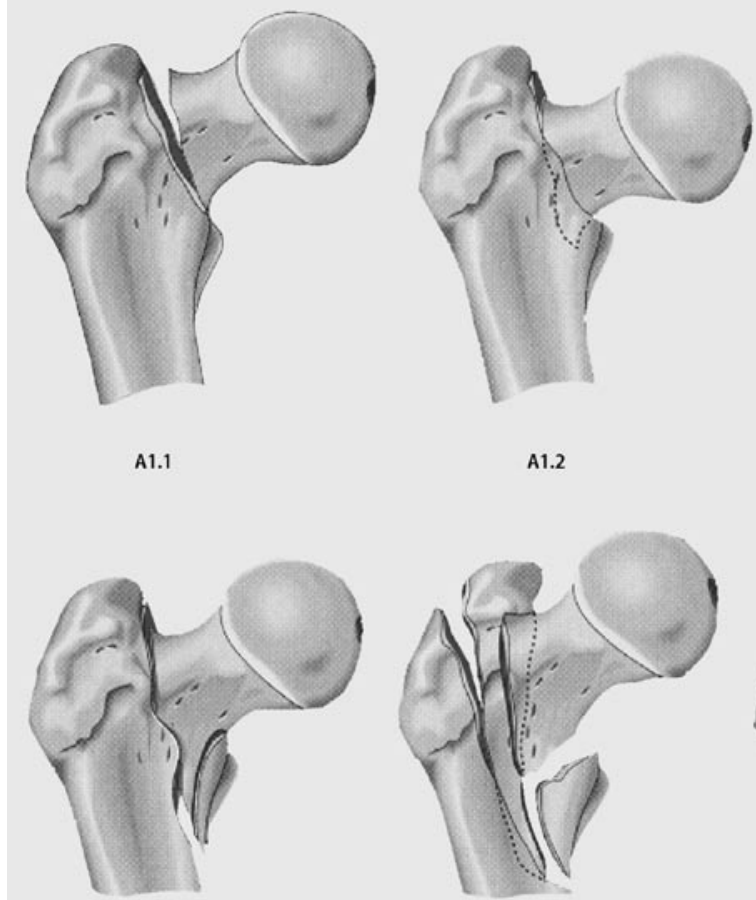

A2.1

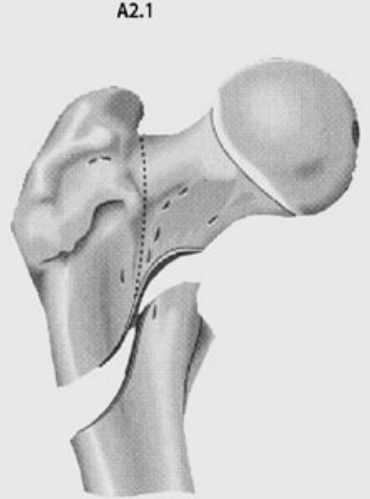

A3.1
A1.2

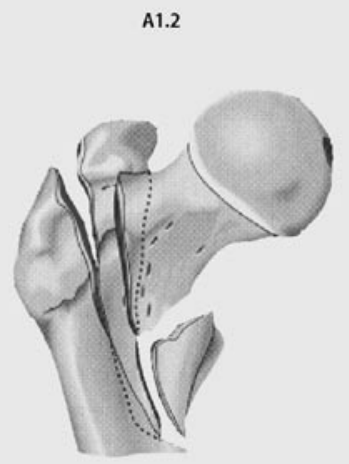

A2.2

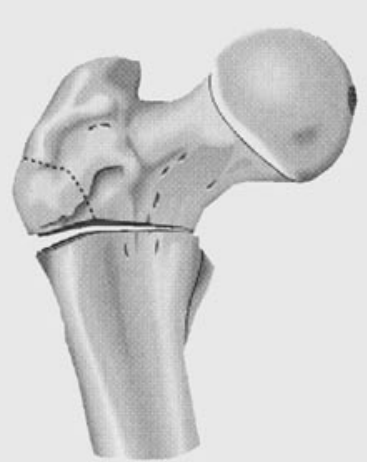

A3.2

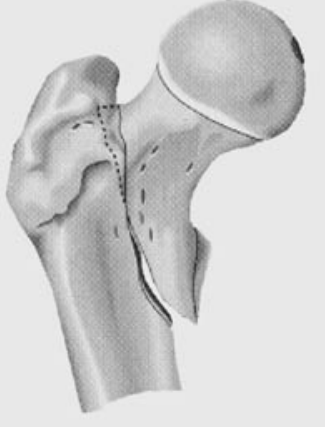

A1.3

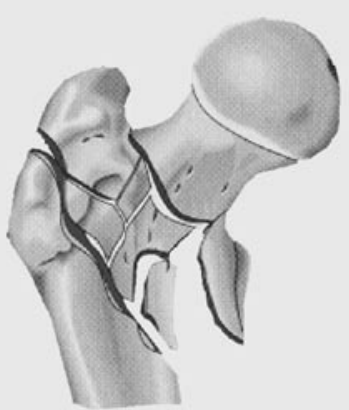

A2.3

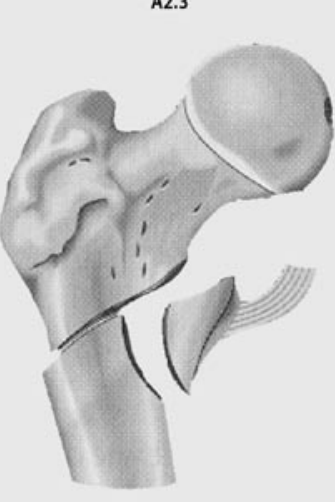

A3.3

Abb. $1 \Delta$ Klassifikation der proximalen Femurfrakturen [AO/ASIF (Association for the Study of internal Fixation)]

\section{Implantat}

Seine Wahl hängt ebenfalls von der Frakturgeometrie und -dynamik ab sowie von der Knochenqualität. Geprüft werden müssen auch die Möglichkeiten der Frakturreposition. Sollte z. B. eine geschlossene Reposition nicht einwandfrei möglich sein, muss dies frühzeitig in die operative Taktik mit einbezogen werden. Gegebenfalls muss auf eine offene Reposition über einen entsprechend größeren operativen Zugang ausgewichen werden.

Bezüglich der Implantate gibt es prinzipiell 3 Möglichkeiten:

- das extramedulläre statische Verfahren,

- das extramedulläre dynamische Verfahren, wie die dynamische Hüftschraube, sowie

- intramedulläre Verfahren, die in der Regel bereits eine dynamische hüftnahe Komponente beinhalten.

Erfahrungen der vergangenen Jahrzehnte haben dazu geführt, dass das extramedulläre statische Verfahren, z. B. in Form einer Winkelplatte, oder die dynamische Kondylenplatte nicht mehr die erste Wahl in der Versorgung einer proximalen $\mathrm{Fe}$ murfraktur sind. Zu häufig ist es entweder zu einem Ausschneiden der im Schenkelhals liegenden Implantatkomponente gekommen oder aber zu einem Implantatbruch in Höhe der Fraktur.

Aktuell wird man sich daher in erster Linie für ein dynamisches Implantat entscheiden. Hier besteht dann die Wahl zwi-
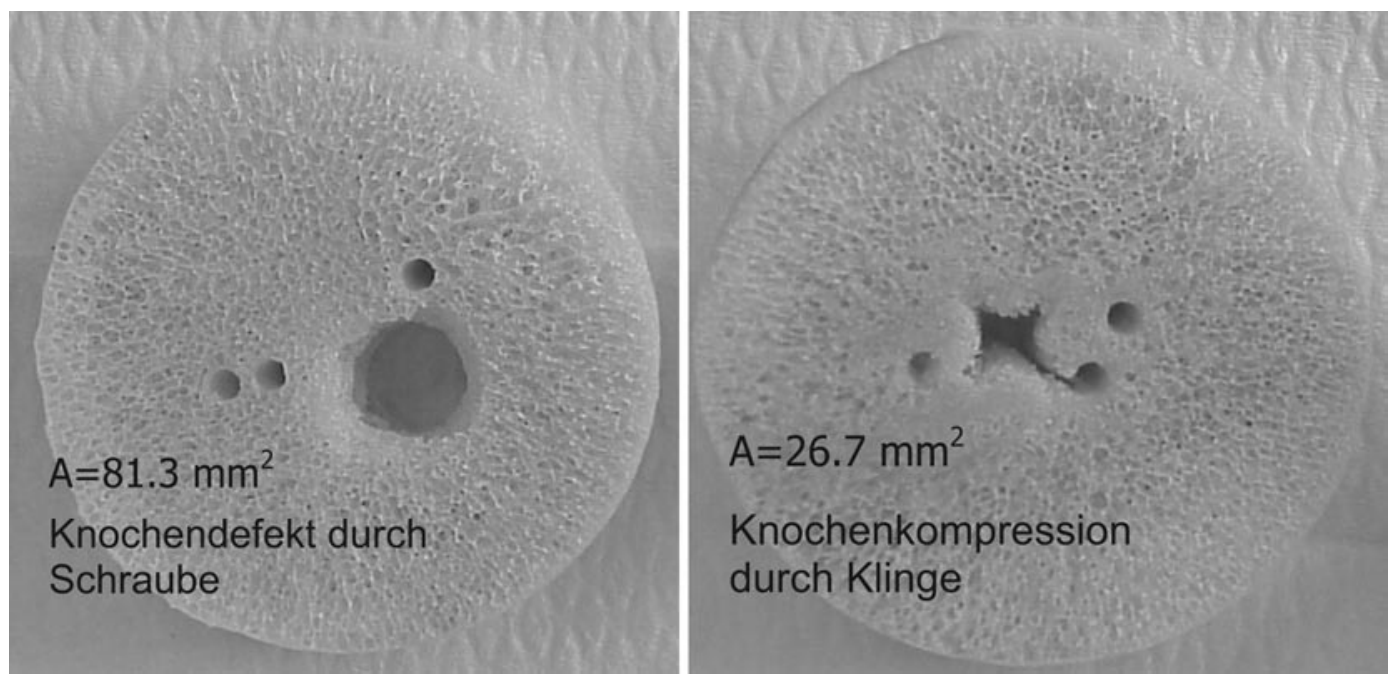

Abb. $2<$ Implantat bedingte Defektgrößen im Femurkopf, a Knochendefekt durch Schraube, Fläche $81,3 \mathrm{~mm}^{2}$, b Knochenkompression durch Klinge, Fläche $26,7 \mathrm{~mm}^{2}$ (AO-Foundation) 
schen einem extra- oder einem intramedullären Implantat.

Bezüglich des Implantatdesigns hat es im Lauf der letzen Jahre ebenfalls eine weitergehende Entwicklung gegeben. So wurde für die dynamische Hüftschraube die so genannte Trochanterabstützplatte entwickelt, die aus Autorensicht aber eher als Salvage-Verfahren zu werten ist und nicht im Rahmen der Standardversorgung eine Rolle spielen sollte.

Die intramedullären Implantate wurden ebenfalls weiterentwickelt. Bei den ersten Implantatgenerationen kam es häufig zur einer Stressfraktur am Ende des Implantats, deshalb wurde inzwischen der intramedulläre Femurschaftanteil etwas über die distale Verriegelungsschraube hinaus verlängert. Des Weiteren wurde die so genannte Schenkelhalskomponente verändert, da durch das Einbringen einer Schraube ein gewisser Knochendefekt verblieb, was gerade beim osteoporotischen Knochen zu einem verminderten Halt des Implantats führte. Als wesentlich vorteilhafter erwies sich das Einbringen einer Klinge, die ohne Vorbohren eingeschlagen wird. Hierdurch kommt es zu einer Knochenkompression in der unmittelbaren Kontaktzone zwischen spongiösem Knochen und dem Implantat selbst

\section{(• Abb. 2).}

Bezüglich der Entscheidung DHS („dynamic hip screw“) oder intramedulläres Implantat gab es eine Reihe von unterschiedlichen Untersuchungen, die letzten Endes zu keinem einheitlichen Ergebnis kamen, sodass sich, bis zum heutigen Tag, nur schwer eine wirklich evidenzbasierte Empfehlung zu dieser Fragestellung geben lässt $[1,2,4,7,8,9,10]$. Auch ein Vergleich verschiedener intramedullärer Implantate ergab keine signifikanten Unterschiede, sodass auch hier keine evidenzbasierte Empfehlung für ein bestimmtes intramedulläres Implantat ausgesprochen werden kann [6]. Eine retrospektive Analyse kam zu dem Schluss, dass letzten Endes die derzeit zur Verfügung stehenden Implantate, im Sinne einer dynamischen Hüftschraube und den intramedullär einzubringenden Implantaten, die einzigen Implantate der Wahl darstellen [11].

Betrachtet man die Literatur kritisch, lässt sich dennoch folgende minimale
Trauma Berufskrankh 2009 • 11[Suppl 1]:107-111

(c) Springer Medizin Verlag 2008

\section{A. Seekamp \\ Fraktur am proximalen Femur}

\section{Zusammenfassung}

Frakturen des proximalen Femurs erfordern in der Regel eine operative Versorgung. Bei der Verfahrenswahl spielt die Klassifikation eine entscheidende Rolle, aber auch die Erwartungen des Patienten sollten berücksichtig werden. In der Regel sollte es diesem ermöglicht werden, möglichst rasch in sein gewohntes Lebensumfeld zurückzukehren und seine vorbestehende körperliche Leistungsfähigkeit wieder zu erreichen. Bezüglich der Verfahrenswahl sind nicht genügend Daten für evidenzbasierte Empfehlungen vorhanden, grundsätzlich aber sollte durch eine operative Versorgung eine Vollbelastung angestrebt werden. Bei einer einfachen pertrochanteren Fraktur ohne Beteiligung des Tro-

\section{Proximal femur fracture}

\section{Abstract}

Proximal femur fractures generally require surgical treatment. Although classification plays an important role in the choice of procedure, patient expectations should also be taken into consideration. In general, a prompt return to everyday life and previous physical condition should be made possible. Insufficient data is available regarding procedure options to make evidence-based recommendations; however, surgical treatment should basically aim to achieve full weight-bearing. In the case of a simple pertrochanteric fracture without involvement of the trochanter
DOI 10.1007/s10039-008-1407-8

chanters minor kann regelhaft ein extramedulläres Implantat gewählt werden. Handelt es sich um eine pertrochantere Fraktur mit Abriss des Trochanter minor oder um einen subtrochanteren Bruch, ist eher ein intramedulläres Implantat zu empfehlen. Bei entsprechender Indikationsstellung und adäquater Wahl des Implantats kann auch eine grob dislozierte proximale Femurfraktur durch eine geschlossene Reposition ausreichend reponiert und erfolgreich zur Ausheilung gebracht werden.

\section{Schlüsselwörter}

Proximaler Femur - Fraktur - Klassifikation . Operative Versorgung $\cdot$ Verfahrenswahl

minor, an extramedullary implant can, as a rule, be chosen. In the case of a pertrochanteric fracture with a torn-away trochanter minor or a subtrochanteric break, an intramedullary implant is recommended. With correct diagnosis and appropriate choice of implant, even a bluntly dislocated proximal femur can be adequately set and successfully healed by means of closed repositioning.

\section{Keywords}

Proximal femur - Fracture - Classification . Surgical treatment $\cdot$ Choice of procedure 


\section{Der vermeintlich einfache Fall}
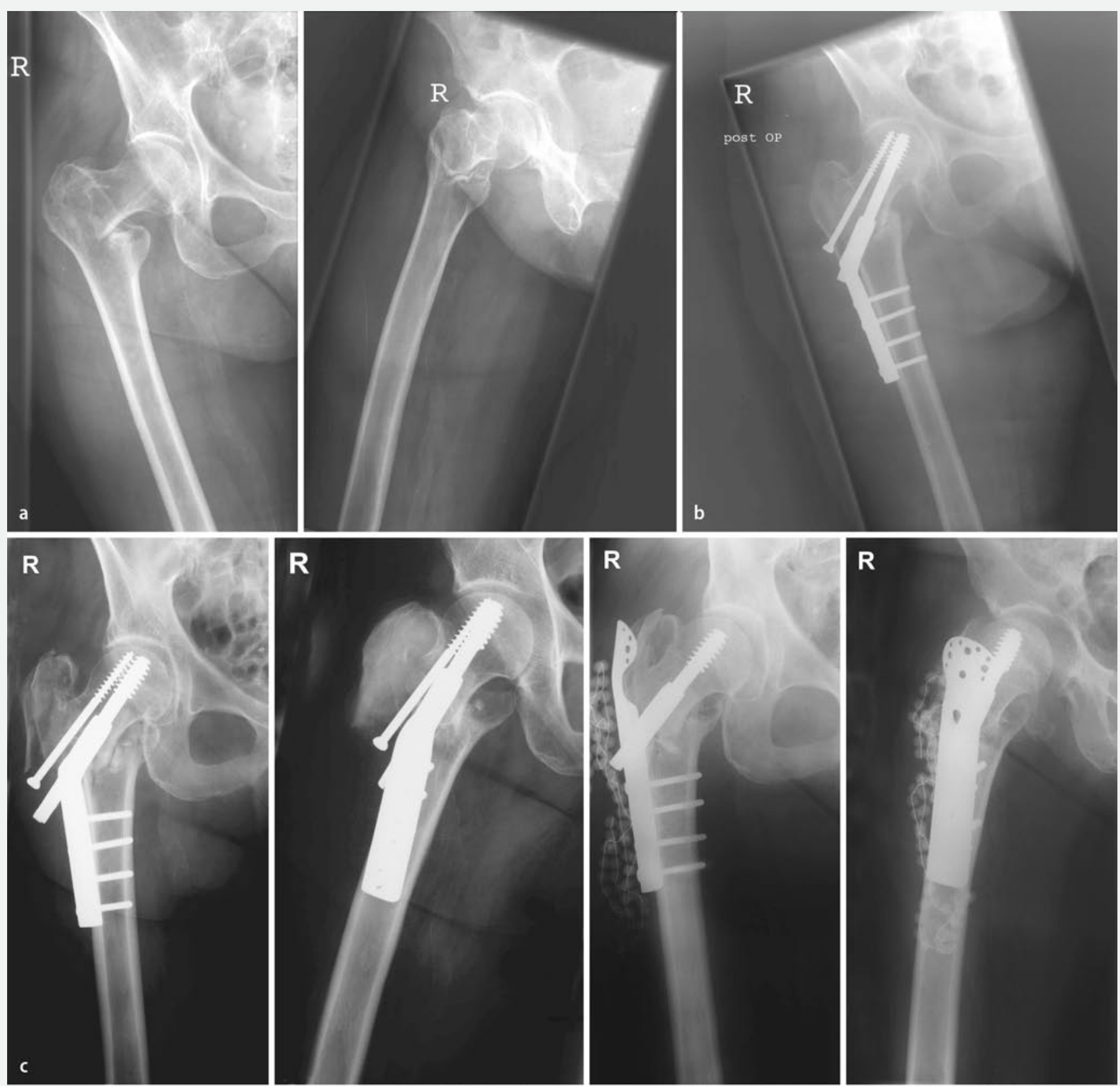

$\mathbf{R}$
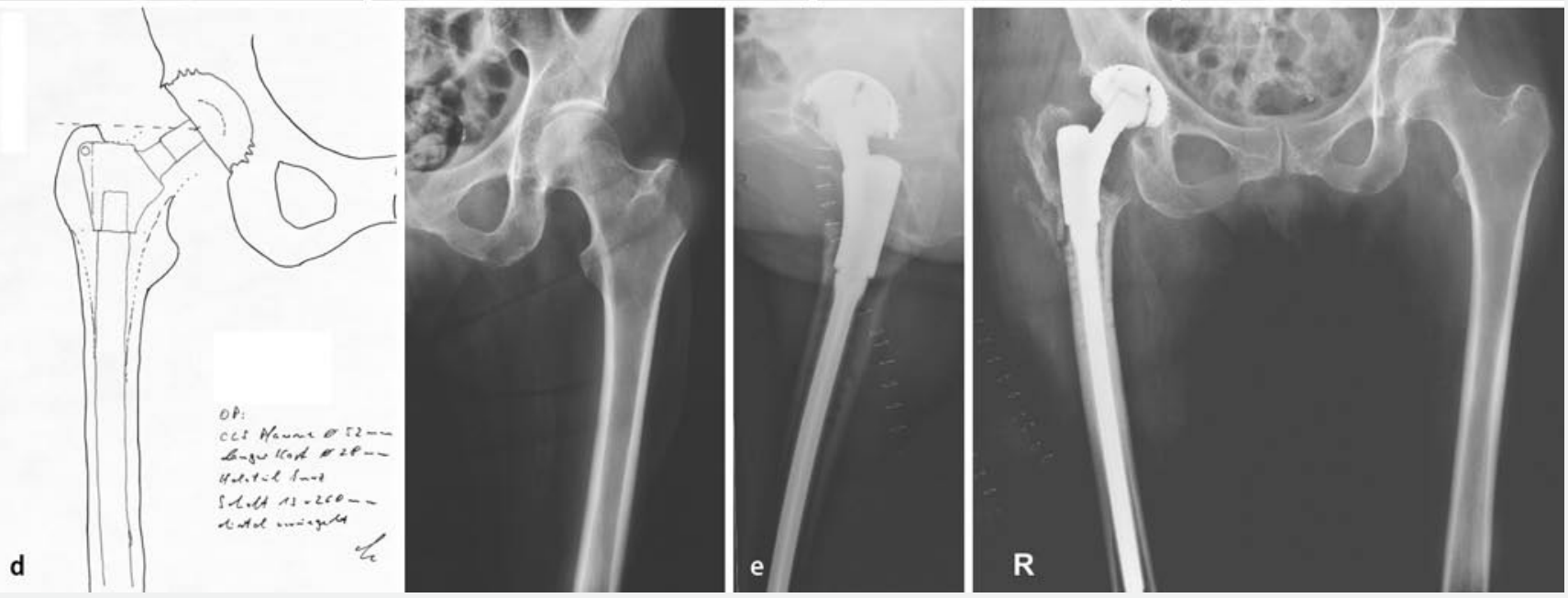
Empfehlung geben. Grundsätzlich sollte, wie schon zuvor angegeben, durch eine operative Versorgung eine Vollbelastung angestrebt werden. Handelt es sich um eine einfache pertrochantere Fraktur ohne Beteiligung des Trochanter minor, kann regelhaft ein extramedulläres Implantat, z. B. die dynamische Hüftschraube, gewählt werden. Dies beinhaltet ohne Weiteres die Möglichkeit der Vollbelastung. Handelt es sich jedoch um eine pertrochantere Fraktur mit Abriss des Trochanter minor, ist eher ein intramedulläres Implantat zu empfehlen. Gleiches gilt für jede Art der subtrachonteren Fraktur. Die Erfahrung hat gezeigt, dass bei adäquater Indikationsstellung und entsprechender Wahl des Implantats auch eine grob dislozierte proximale Femurfraktur durch eine geschlossene Reposition ausreichend reponiert und erfolgreich zur Ausheilung gebracht werden kann, selbst wenn primär in Teilen noch eine grobe Dislokation der Fragmente zueinander verblieben ist. Dies gilt für jüngere ebenso wie für ältere Patienten. Insbesondere hat sich auch erwiesen, dass gerade der Trochanter minor selbst bei gröberer Dislokation wieder einheilt (• Abb. 3).

\section{Fallbeispiel}

Abschließend sei noch eine pertrochantere Fraktur dargestellt, die primär als einfache Fraktur eingeschätzt wurde und, wie auch hier empfohlen, bei nicht frakturiertem Trochanter minor mit einer dynamischen Hüftschraube versorgt wurde (• Abb. 3).

Im Anschluss kam es unter der Mobilisierung unter halber Körpergewichtsbelastung zu einer erneuten Dislokation der

Abb. 34 Fallbeispiel einer pertrochanteren Femurfraktur mit kompliziertem Verlauf, a Unfallaufnahmen, $\mathbf{b}$ primäre Stabilisierung durch dynamische Hüftschraube, $\mathbf{c}$ weiterer postoperativer Verlauf: nach Mobilisierung sekundäre Dislokation der versorgten pertrochanteren Femurfraktur und der Implantate, Verdacht auf Infekt, Reosteosynthese durch DHS kombiniert mit Abstützplatte, postoperativer Nachweis von Staphylococcus aureus, mehrere Revisionsoperationen, $\mathbf{d}$ Planung eines prothetischen Ersatzes des Hüftgelenks mit gespiegelter Gegenseite als Planungsreferenz, e postoperative Aufnahmen, weitere Erläuterungen s. Text
Fraktur und der Implantate mit Medialisierung des Femurschafts im Verlauf von 6 Wochen. Es wurde versucht, diese Situation durch zusätzliches Einbringen einer Trochanterabstützplatte über die DHS 7 Wochen nach der Erstversorgung zu retten. Postoperativ wurden Staphylococcus aureus nachgewiesen und mehrere Revisionen mit Einlage von Antibiotikaketten durchgeführt. Letzten Endes war dieser Rettungsversuch jedoch nicht erfolgreich. Es blieben nur die komplette Implantatentfernung und, bei gleichzeitig eingetretener Subluxation des Hüftgelenks, anschließend der komplette Hüftgelenkersatz mit einer Revisionsprothese.

Retrospektiv gesehen ist entweder die Fraktur initial nicht in ihrem Ausmaß erkannt worden oder aber es ist in der Tat zweitseitig, unter der Mobilisierung, zu einer Frakturausdehnung gekommen, sodass das gesamte Trochantermassiv instabil wurde.

\section{Fazit}

Um den geschilderten oder ähnlich komplizierte Verläufe von vornherein zu vermeiden und dass der vermeintlich einfache Fall tatsächlich ein einfacher Fall bleibt, lässt sich abschließend Folgendes empfehlen. Wichtig erscheint eine adäquate Klassifikation, wobei insbesondere das Kriterium der medialen Abstützung beachtet werden sollte. Des Weiteren gilt, dass vorzugsweise eine intramedulläre Stabilisierung gewählt werden sollte, je höhergradiger die Instabilität der Fraktur eingeschätzt wird. Schließlich erscheint es von Bedeutung, dass eine adäquate Reposition erzielt wird. Dies spielt gerade bei instabilen Frakturen eine entscheidende Rolle. Wird ein intramedulläres Implantat gewählt, muss gewährleistet sein, dass dieses im distalen Fragment auch einen entsprechenden Halt findet. Gegebenenfalls ist hierzu eine offene Reposition des Bruchs mit Adaptation insbesondere der Femurschaftanteile erforderlich, die durch zusätzliches Einbringen einer Cerclage gehalten werden können. Unter Beachtung dieser 3 Empfehlungen sollte eine adäquate Versorgung jeglicher Frakturen des proximalen Femurs gut gelingen.

\section{Korrespondenzadresse}

Prof. Dr. A. Seekamp

Klinik für Unfallchirurgie (Traumatologie), Universitätsklinikum Schleswig-Holstein, Campus Kiel,

Arnold Heller-Straße 7, 24105 Kiel

uta.hansen@uksh-kiel.de

Interessenkonflikt. Keine Angaben.

\section{Literatur}

1. Al-Yassari G, Langstaff RJ, Jones JWM et al. (2002) The AO/ASIF proximal femur nail (PFN) for treatment of unstable trochanteric femoral fracture. Injury 33: 395-399

2. Audige L, Hanson B, Swiontkowski MF (2003) Implant-related complications in the treatment of unstable intertrochanteric fractures: meta-analysis of dynamic screw-plate versus dynamic screw-intramedullary nail devices. Int Orthop 27: 197-203

3. Evans E (1949) Pronation injuries of the forearm, with special reference to the anterior Monteggia fracture. J Bone Joint Surg Br 31: 190-203

4. Harrington $P$, Nihal A, Singhania AK et al. (2002) Intramedullary hip screw versus sliding hip screw for unstable intertrochanteric femoral fractures in the elderly. Injury 33 : 23-28

5. Jensen JS, Michaelsen M (1975) Trochanteric femoral fractures treated with McLaughlin osteosynthesis. Acta Orthop Scand 46: 795-803

6. Müller M, Seitz A, Besch L et al. (2008) Klinik für Unfallchirurgie, Universitätsklinikum SchleswigHolstein, Campus Kiel, Versorgung pertrochantärer Femurfrakturen, Vergleichende Analyse mit PFN vs. TGN „,keine signifikanten Unterschiede“. Unfallchirurg 111: 71-78

7. Nuber S, Schönweiss T, Rüter A (2003) Stabilisierung von instabilen trochantären Mehrfragmentfrakturen, Vergleich zwischen PFN und DHS mit Trochanterabstützplatte. Unfallchirurg 106: 39-47

8. Parker MJ, Gillespie LD, Gillespie WJ (2001) Hip protectors for preventing hip fractures in the elderly. Cochrane Database Syst Rev 3: CD001255

9. Preite R, Belli PG, Giordano MC et al. (2000) A comparative analysis of the different fixation devices used in pertrochanteric fractures. Chir Organi Mov 85: 225-233

10. Saudan M, Lubbeke A, Sadowski C et al. (2002) Per trochanteric fractures: is there an advantage to an intramedullary nail? A randomized, prospective study of 206 patients comparing the dynamic hip screw and proximal femoral nail. J Orthop Trauma 16: 386-393

11. Stern $R$ (2007) Are there advances in the treatment of extracapsular hip fractures in the elderly? Injury [Suppl 3] 38: S77-87 